\title{
PanDrugs: a novel method to prioritize anticancer drug treatments according to individual genomic data
}

Elena Piñeiro-Yáñez ${ }^{1 \dagger}$, Miguel Reboiro-Jato ${ }^{2,3 \dagger}$, Gonzalo Gómez-López ${ }^{1}$, Javier Perales-Patón ${ }^{1}$, Kevin Troulé1, José Manuel Rodríguez ${ }^{4}$, Héctor Tejero ${ }^{1}$, Takeshi Shimamura ${ }^{6}$, Pedro Pablo López-Casas ${ }^{1}$, Julián Carretero ${ }^{5}$, Alfonso Valencia', Manuel Hidalgo ${ }^{1,7}$, Daniel Glez-Peña ${ }^{2,3}$ and Fátima Al-Shahrour ${ }^{1 *}$ (D)

\begin{abstract}
Background: Large-sequencing cancer genome projects have shown that tumors have thousands of molecular alterations and their frequency is highly heterogeneous. In such scenarios, physicians and oncologists routinely face lists of cancer genomic alterations where only a minority of them are relevant biomarkers to drive clinical decisionmaking. For this reason, the medical community agrees on the urgent need of methodologies to establish the relevance of tumor alterations, assisting in genomic profile interpretation, and, more importantly, to prioritize those that could be clinically actionable for cancer therapy.

Results: We present PanDrugs, a new computational methodology to guide the selection of personalized treatments in cancer patients using the variant lists provided by genome-wide sequencing analyses. PanDrugs offers the largest database of drug-target associations available from well-known targeted therapies to preclinical drugs. Scoring datadriven gene cancer relevance and drug feasibility PanDrugs interprets genomic alterations and provides a prioritized evidence-based list of anticancer therapies. Our tool represents the first drug prescription strategy applying a rational based on pathway context, multi-gene markers impact and information provided by functional experiments. Our approach has been systematically applied to TCGA patients and successfully validated in a cancer case study with a xenograft mouse model demonstrating its utility.

Conclusions: PanDrugs is a feasible method to identify potentially druggable molecular alterations and prioritize drugs to facilitate the interpretation of genomic landscape and clinical decision-making in cancer patients. Our approach expands the search of druggable genomic alterations from the concept of cancer driver genes to the druggable pathway context extending anticancer therapeutic options beyond already known cancer genes. The methodology is public and easily integratable with custom pipelines through its programmatic API or its docker image. The PanDrugs webtool is freely accessible at http://www.pandrugs.org.
\end{abstract}

Keywords: Precision oncology, Personalized medicine, Translational bioinformatics, Cancer genomics, In silico prescription, Targeted therapy, Druggable genome

\footnotetext{
* Correspondence: falshahrour@cnio.es

†Elena Piñeiro-Yáñez and Miguel Reboiro-Jato contributed equally to this work.

${ }^{1}$ Spanish National Cancer Research Centre (CNIO), 3rd Melchor Fernandez

Almagro st., E-28029 Madrid, Spain

Full list of author information is available at the end of the article
}

(c) The Author(s). 2018 Open Access This article is distributed under the terms of the Creative Commons Attribution 4.0 International License (http://creativecommons.org/licenses/by/4.0/), which permits unrestricted use, distribution, and reproduction in any medium, provided you give appropriate credit to the original author(s) and the source, provide a link to the Creative Commons license, and indicate if changes were made. The Creative Commons Public Domain Dedication waiver (http://creativecommons.org/publicdomain/zero/1.0/) applies to the data made available in this article, unless otherwise stated. 


\section{Background}

Identifying the most appropriate therapies from cancer genome data is a major challenge in personalized cancer medicine. Standard of care treatments are commonly selected following criteria such as: cancer type; stage; patient status; and/or the presence of prognostic and predictive biomarkers. However, cancer treatment could be revolutionized if the information contained in large genomic datasets were to be systematically deconvoluted in terms of potential treatments [1]. Here, the identification and evaluation of somatic alterations and their collective impact on tumor progression pose considerable challenges to their clinical application [2, 3]. More specifically, physicians and researchers are challenged with long lists of tumor-specific genomic variants where most variants are either clinically "unactionable," their biological role unknown, or they are irrelevant for tumor biology [4]. In addition, the current list of cancer driver genes [5] has clinical limitations since genomic alterations in these genes may be essential for oncogenesis, tumor cell growth, and survival; but the same genes may not be targetable by current therapies [6]. Moreover, very recent studies have revealed that cancer gene lists are still incomplete and that there are many more cancer genes yet to be discovered [7-9]. In this scenario, it is essential to develop new methodologies to analyze genetic alterations in terms of treatment options, helping to prioritize those that could be useful for the management of cancer patients.

Several remarkable efforts have addressed the prioritization of genomic alterations [10-13]. These methods exploit the extensive literature and knowledge available in public repositories to catalogue cancer genomic variants and their impact on biological functions, although none of these methodologies directly link genomic alterations to potential therapies. Tools such DGIdb [14], OncoKB [15], and the Cancer Genome Interpreter (CGI) [16] have been developed to identify clinically actionable genomic alterations in tumors. Although these tools demonstrate the potential of targeted therapies and provide drug repurposing strategies, they present some limitations. They only consider known cancer driver genes for drug prescription, they are based exclusively on somatic DNA alterations, the therapeutic options are restricted to "one target - one drug" ignoring multiple targetable mutations and the protein pathway-specific activity, and they do not provide a prioritized list of treatments based on clinical, biological, and pharmacological evidence.

Here we introduce PanDrugs, a new computational resource to propose drug therapies from genome-wide experimental results, including variant and gene lists. PanDrugs expands cancer therapeutic options by taking into account multiple genomic events potentially responsive to a treatment, the pathway context [17], and the pharmacological evidence reported in large-scale experiments $[18,19]$.

\section{Implementation \\ PanDrugs database}

The PanDrugs database (PanDrugsdb) has been implemented to store gene-drug relationships. PanDrugs methodology mines PanDrugsdb to provide a catalogue of prioritized candidate drugs and targetable genes estimated from a list of variants (or genes) provided by a user (Fig. 1a).

Pharmacological data and drug annotations available in PanDrugsdb were collected from 24 databases. These included 18 sources with information curated by experts and gene-drug associations obtained from experimental drug screenings: The Cancer Therapeutics Response Portal [19] and GDSC [20] (Additional file 1: Table S1). Since different sources employ a variety of non-standardized identifiers to mention the same compound, drug names were standardized in order to be consistently integrated within PanDrugsdb (Additional file 1: Materials and Methods). Following this, drug annotations were enriched with additional information regarding drug families, drug indication status, cancer type, and therapy description (Additional file 1: Figure S1 and S2). Gene-drug relationships were also annotated with the type of gene-drug relation (i.e. drug target or biomarker), drug sensitivity or resistance response, and the type of genomic alteration associated to the drug response.

The current version of PanDrugsdb includes 9092 drugs, 4804 unique genes, and 43,909 direct and non-redundant gene-drug interactions. The database is built in MySQL RDBMS. Full details regarding PanDrugsdb implementations and PanDrugs software are described in Additional file 1: Materials and Methods.

\section{PanDrugs nomenclature}

PanDrugs categorizes druggable genes as: (1) direct targets; (2) biomarkers; and (3) pathway members.

The term "direct targets" includes those genes that contribute to disease phenotype and can be directly targeted by a drug (i.e. small molecule or monoclonal antibody). For instance, $B R A F$ is a direct target for vemurafenib [21]. "Biomarkers" refers to genes that have a genetic status associated with drug response (according to clinical or pre-clinical evidence) but the protein product is not the drug target itself. For example, BRCA-mutated cancers that respond to poly-ADP-ribose polymerase $(P A R P)$ inhibitors [22], PTEN loss that is associated with decreased sensitivity of colorectal cancer tumors to anti-EGFR antibodies [23], or mutations in TSC1/2 as clinically approved biomarkers of PI3K/Akt/mTOR inhibitor response $[24,25]$. PanDrugs "biomarkers" information was obtained from manually curated databases (see Additional file 1: 


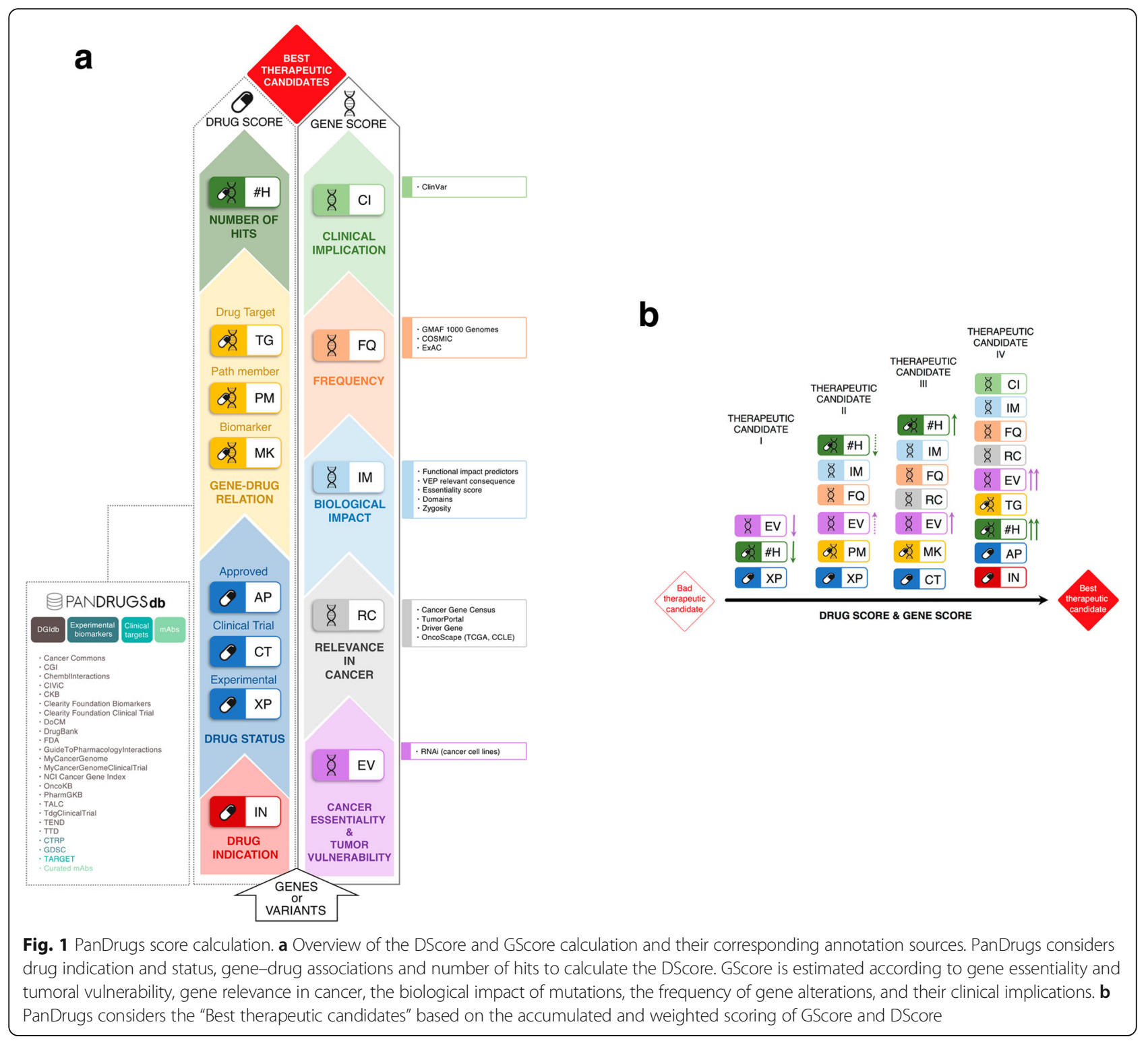

Materials and Methods for details) and from experimental assays in cancer cell lines (GDSC and CTRP).

Targeted therapies may target cell signals that are needed for cancer cells to develop, proliferate, and invade. Drugs targeting the activity of the surrounding interactors in the biological pathway of a mutated gene could: (1) produce the same downstream effect as targeting the mutated gene itself; (2) enhance response by synergistic effects; and (3) be used in combination to avoid possible compensatory drug resistance mechanisms [26-29]. Following this paradigm, PanDrugs includes "pathway member" referring to all those downstream druggable targets taking advantage of the pathway background underlying the user's gene list. Interestingly, this paradigm unlocks alternative therapeutic ways for untargetable genes.
Finally, PanDrugs analyzes the "collective gene impact" defined as the number of druggable genes (direct targets, biomarkers, and pathway members) in the input list that points to a particular drug. Those drugs capable of targeting the largest number of druggable genes are prioritized.

PanDrugs uses two scores to prioritize cancer treatments PanDrugs calculates two scores integrating a variety of clinical, biological, and pharmacological sources and databases to suggest tailored anticancer therapies based on user supplied variant lists and PanDrugsdb (Fig. 1a). Gene Score (GScore) is in the range of $0-1$ based on the level of evidence supporting gene clinical implication and its biological relevance in cancer (Additional file 1: Figure S3A). Drug Score (DScore) estimates drug response and treatment suitability (Additional file 1: Figure 
S3B). A larger number of supporting databases, curated annotation, and clinical impact enhance the weight in both GScore and DScore calculation. Full descriptions of GScore and DScore calculations are available in Additional file 1: Materials and Methods.

GScore has been implemented to consider: (1) genomic feature evidence by mutation consequence, functional impact, and population allele frequency; (2) relevance in cancer estimated by Cancer Gene Census (CGC) of COSMIC v84 [30], TumorPortal resource [31], Tamborero et al. [32], and OncoScape [33]; (3) essentiality from RNA interference (RNAi) experiments in cancer cell lines from Achilles project [34, 35] and; (4) clinical implications based on its pathogenicity supporting evidence (taken from COSMIC and ClinVar). GScore weight assignation for non-ranked gene lists and for VCF files are described in Additional file 1: Tables S2 and $\mathrm{S} 3$, respectively.

DScore is calculated using PanDrugsdb to evaluate the therapeutic implications of those altered genes previously employed for GScore calculation. DScore takes into account: (1) drug-cancer type indication (from the FDA and clinicaltrials.gov); (2) drug clinical status (approved by the FDA, clinical trials, or preclinical); (3) gene-drug relationship (i.e. direct target, biomarker, or pathway member); (4) number of curated databases supporting that relationship (i.e. database factor); and (5) collective gene impact (Additional file 1: Figure S3C). DScore has values from -1 to 1 where negative values correspond to drug unresponsiveness and positive values to drug sensitivity (Additional file 1: Figure S3B).

PanDrugs provides a prioritized list of candidate drugs considering GScore and DScore values. Those drug therapies supported by scores nearest to 1 in both GScore and DScore will have more evidence for their effectiveness in cancer treatment and will be considered "Best therapeutic candidates" by PanDrugs (Fig. 1b).

\section{Exploiting pathway information to increase therapeutic options}

PanDrugs expands the anticancer therapeutic arsenal suggesting drugs to target genes located downstream to the altered gene(s). To this end, PanDrugs integrates previously modelled biological circuit information (e.g. signaling pathways) [36], the interaction types between nodes (activation or inhibition), and the gene functional role (oncogene or tumor suppressor). Ideally, a perfect gene-drug(s) solution will meet the following criteria: (1) the gene is affected by activating point mutations (predicted by functional impact algorithms or confirmed by databases/literature); (2) the gene is essential in synthetic lethal screenings; (3) the gene is sensitive to specific drugs included in PanDrugs; and (4) an FDA drug is approved or under clinical trial that targets the gene.
Although it is known that only few genomic alterations will follow these stringent criteria, the emerging "druggable genome" concept opens the whole genome to therapeutic intervention. In other words, both a given mutated gene and its interactions are putative drug targets [37, 38]. Following this paradigm, PanDrugs offers a systems biology framework to propose drugs that arise as rational therapeutic candidates. For example, $M E T$ amplification plays a role in acquired resistance to EGFR inhibitors of patients with EGFR-mutated tumors by activating MAPK and PI3K/AKT signaling pathways [39, 40]. Combination therapy of EGFR and MET inhibitors is used to block both MET and EGFR signaling pathways [41]. In this scenario, PanDrugs proposes the following as therapeutic options: (1) avoiding EGFR inhibitors alone due to the known lack of sensitivity; (2) the usage of MET inhibitors that can overcome resistance of EGFR-TKIs; and (3) targeting downstream druggable genes (i.e. $R A F, M E K$ ) with available drugs (i.e. Sorafenib, Trametinib) blocking the oncogenic consequences of the pathway overstimulation [42, 43] (Fig. 2).

\section{PanDrugs web tool and application programming interface} PanDrugs is available as a user-friendly web tool with preloaded queries and demo examples at http://www.pandrugs.org. The detailed user manual is accessible online at https://www.pandrugs.org/\#!/help. The server supports three alternative types of input: (1) single and multiple queries (gene lists); (2) standard VCF files; and (3) a ranked list of genes, where ranking is obtained from experimental observations (i.e. RNA-sequencing experiments). Regular analysis of a 500-line VCF file takes an average time of $\sim 6 \mathrm{~min}$ in the PanDrugs server. The results page provides a panel with basic statistics of the query, pie-charts depicting clinical status distribution and families for the drugs proposed, and a bubble plot representing GScore and DScore together with the best candidate therapies suggested by PanDrugs. Moreover, the tool generates a ranked summary table of the treatments with raw scores and links to external databases (Additional file 1: Figure S4). In addition to GScore and DScore, the summary table displays comprehensive and sortable information about genes, drugs, type of genedrug interaction, drug family, drug clinical status, type of therapy available (only for the approved drugs), and sources of annotation employed. PanDrugs uses KEGG pathways to map the relationship between input genes and pathway members suggested as candidate drug targets. Our tool also supports drug queries to explore the gene-drug interactions available in PanDrugsdb providing the subsequent summary table. All results generated by PanDrugs are easily downloadable in standard formats (i.e. CSV, PNG, PDF, etc.). PanDrugs provides a REST-based Application Programming Interface (API) allowing developers to make queries directly to PanDrugsdb, to incorporate output from 


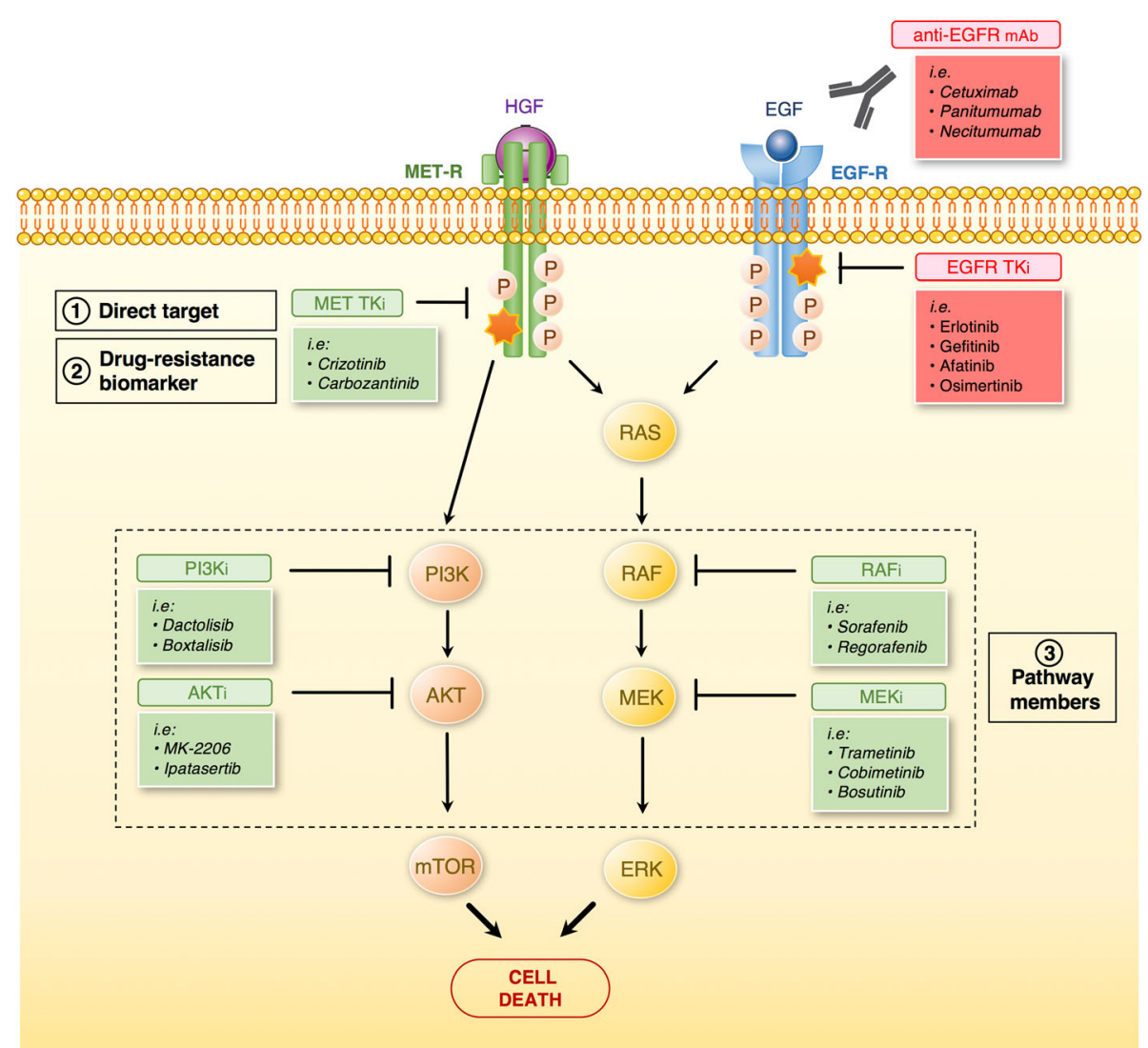

Fig. 2 Possible scenarios for PanDrugs therapeutic candidates. PanDrugs proposes three potential types of druggable candidates. This includes: (1) direct targets, a gene that contributes to a disease phenotype and can be directly targeted by a drug; (2) drug-resistance biomarkers, a gene which genetic status is associated with a drug response from clinical or pre-clinical evidence but its protein product is not the direct target of the drug; and (3) pathway members, a targetable gene located downstream to the altered one. To illustrate this, tumors mutated in EGFR carrying MET amplifications will not respond to EGFR inhibitors (red). PanDrugs proposes as therapeutic strategy MET inhibitors and targeting MET downstream proteins (green) to drive tumor cell death

PanDrugs within their own algorithms, and to combine the tool as a novel module in NGS analysis pipelines integrating genetic data and therapeutic alternatives. PanDrugs is also available as a docker image at https:/github.com/ sing-group/pandrugs-docker.

\section{Results}

\section{PanDrugs in The Cancer Genome Atlas (TCGA) data}

PanDrugs has been systematically applied to a cohort of 7069 samples from the TCGA project that correspond to 20 different tumor types (Additional file 1: Figure S5). File sources employed for the TCGA analysis are listed in Additional file 1: Table S4. Databases used in the study and their corresponding versions are detailed in Additional file 1: Table S5. Full results may be interactively accessed at the PanDrugs website (http:// www.pandrugs.org/).

The PanDrugs analysis of TCGA tumors showed that the GScore distribution of genes affected by genomic alteration events (SNVs + indels + Copy Number Variations (CNVs)) drops drastically at GScore $=0.4$. In agreement with previous studies, this observation suggests that most genomic alterations in TCGA patients have little evidence of being associated with cancer and, in consequence, are poorly annotated in public databases [31, 44] (Additional file 1: Figure S6A). Genes with a GScore $>0.4$ and carrying at least one mutational and/or CNV event were used to identify potential therapies and were found present in $>6000$ of the 7069 TCGA samples (Additional file 1: Figure S6B, S6C; Table S6). We decided to use this threshold for TCGA analysis to establish a compromise solution between gene annotation quality and retaining the largest number of patients. For instance, EGFR mutations across different TCGA tumor types exhibit a $0.23<$ GScore $<0.82$ while mutations in KRAS have GScore values in the range of $0.36-0.97$. These GScores underline the biological relevance and clinical utility of both KRAS and $E G F R$ genomic alterations in cancer. Differences in GScore values reflect the higher frequency of KRAS hotspot mutations and their well-known pathogenicity in 
contrast to the EGFR mutational heterogeneity and its broader functional impact and pathological diversity (Additional file 1: Figure S7). In particular, $E G F R_{\mathrm{GScore}}$ $\sim 0.8$ reveals those well-known mutations with clinical significance related to drug response (L858R) while $E G F R_{\mathrm{GScore}} \sim 0.4$ corresponds to mutations residues that are not located in the protein kinase domain and that are less frequently found in cancer patients but that may have a deleterious functional effect (i.e. EGFR p.L62R). $K R A S_{\mathrm{GScore}} \sim 0.9$ represents well-described and very frequent driver mutations located in exon 2 codons 12 and 13 with clinical significance as diagnostic, prognostic, and predictive biomarkers.

Using a GScore threshold our observations showed that an in silico prescription of approved drugs for direct targets plus biomarkers offered treatments for $65 \%$ of patients when point mutations, indels, and $\mathrm{CNVs}$ are considered simultaneously. (Additional file 1: Figure S8A). Notably, PanDrugs is able to extend drug prescription for $86 \%$ of TCGA patients by exploiting pathway memberdrug associations (Additional file 1: Figure S8B).

The PanDrugs TCGA analysis was also used to evaluate the potential of non-driver genes as effective targets for cancer treatment by selecting the most frequently altered genes in TCGA patients annotated in PanDrugsdb. This selection was carried out for every TCGA tumor type by considering the top five genes according to frequency in small variants (point mutations and indels) and CNVs separately. Following these criteria, we obtained 200 alterations located in 100 genes (Additional file 1: Table S7). Interestingly, $54 \%$ of these most frequently altered genes were found druggable but are currently labelled as non-driver genes [45, 32]. Our results strongly suggest that the extension of genomic events detection beyond the known cancer driver genes can help in finding additional effective therapies for cancer treatment.

We compared PanDrugs' performance by applying our methodology to the TCGA cohort previously used in other studies [46, 47] This comparative TCGA analysis showed that the PanDrugs pathway member approach expands therapeutic options for FDA-approved drugs to an average percentage of $93.41 \%$ in TCGA patients (Additional file 1: Figure S9). This result shows that the PanDrugs pathway member strategy might be useful to complement current in silico prescription tools.

\section{Comparison with other tools}

We compared PanDrugs' performance to DGIdb, OncoKB, CGI, CancerResource (CR) [48], Personalized Cancer Therapy (PCT, https://pct.mdanderson.org/), JAX-Clinical Knowledgebase [49], and Precision Medicine Knowledgebase (https://pmkb.weill.cornell.edu/). The tools included in the comparison and the description of their main functionalities are shown in Fig. 3a.
Gene-drug associations per database are compared in Fig. 3b. PanDrugs supports single and multiple queries (i.e. gene lists) as well as standard VCF files, while DGIdb, CR, and PCT do not support variants and OncoKB, PCT, and CR do not accept multiple gene queries. To make tool comparisons viable, we selected as input only those altered genes annotated in cBioPortal.

The comparison was carried out by selecting an EGFR-mutant lung adenocarcinoma patient with known drug-resistance mechanisms to EGFR inhibitors via $M E T$ amplifications from the TCGA cohort (NSCLC, TCGA-38-4629). Our results show that only PanDrugs and CGI alert of the risk of a possible resistance mechanism to EGFR inhibitors. By contrast, the other tools offer EGFR inhibitors as main therapeutic options since they do not support co-occurring alterations among their functionalities.

Remarkably, only PanDrugs suggests clinically approved treatments and drugs in clinical trials for genes not considered by the other tools using biomarkers and pathway members. For instance, PanDrugs prescribes Palbociclib (Additional file 1: Figure S10A), a selective inhibitor of the cyclin-dependent kinases CDK4 and CDK6, to treat this NSCLC patient as a result of the following evidence: (1) CDK6 is a direct target; (2) CCND1, CDKN2A, and CDKN2B are biomarkers; and (iii) CDK4 is a downstream pathway member gene. Another clear example is Navitoclax, a BCL2 family inhibitor currently tested in clinical trials for NSCLC (NCT02520778). PanDrugs suggests Navitoclax since CDK6 is a biomarker of Navitoclax response and BCL2 as pathway member because it is downstream to the TP53 and ERBB2 genes which are altered in this particular NSCLC patient.

Interestingly, PanDrugs is also capable of expanding drug prescription beyond known cancer genes. To illustrate this, we use the list of novel candidate cancer genes provided by Martincorena et al. PanDrugs analysis revealed 436 gene-drug associations not reported by the other tools (i.e. MAP2K7-Lenalidomide, BMPR2-Serdemetan, or ZFP36L2-Embelin) (Additional file 1: Figure S10B). As expected, all these associations have low GScores due to the limited clinical and biological gene annotations; however, 32 associations corresponding to 13 non-driver genes showed DScore $>0.7$, suggesting their viability as potential targets for cancer treatment.

PanDrugs has been integrated within an online resource for PanCancer Analysis of Whole Genomes (PCAWG) covering 2658 donors from 48 cancer types [50]. Among these donors, we chose three patients without druggable cancer driver-altered genes from colorectal, breast, and prostate cancer to evaluate PanDrugs therapeutic proposals. The colorectal cancer patient (DO10486) showed 32 predicted damaged genes. None of the six altered drivers (STAT3, SOX9, ARID1A, TGFBR2, RTN4, PPP2R1A) are 


\begin{tabular}{|c|c|c|c|c|c|c|c|c|c|c|c|}
\hline Resource & Input type & Scope & $\begin{array}{c}\text { Variant } \\
\text { info }\end{array}$ & API & Docker & Multi-query & $\begin{array}{c}\text { Drug } \\
\text { prioritization } \\
\text { score }\end{array}$ & $\begin{array}{c}\text { Gene } \\
\text { prioritization } \\
\text { score }\end{array}$ & $\begin{array}{c}\text { Collective } \\
\text { gene } \\
\text { impact }\end{array}$ & $\begin{array}{l}\text { Pathway } \\
\text { member } \\
\text { approach }\end{array}$ & $\begin{array}{c}\text { Experimental } \\
\text { validation data } \\
\text { integrated }\end{array}$ \\
\hline PanDrugs & Gene, Variant & Genome-wide & $\checkmark$ & $\checkmark$ & $\checkmark$ & $\checkmark$ & $\checkmark$ & $\checkmark$ & $\checkmark$ & $\checkmark$ & $\checkmark$ \\
\hline Cancer Genome Interpreter & Variant & $\begin{array}{l}\text { Only known cancer } \\
\text { genes }\end{array}$ & $\checkmark$ & $\checkmark$ & $x$ & $\checkmark$ & $x$ & $x$ & $\checkmark$ & $x$ & $x$ \\
\hline DGIdb & Gene & Genome-wide & $x$ & $\checkmark$ & $\checkmark$ & $\checkmark$ & $\checkmark$ & $x$ & $x$ & $x$ & $x$ \\
\hline OncokB & Gene, Variant & $\begin{array}{l}476 \text { known cancer } \\
\text { genes }\end{array}$ & $\checkmark$ & $\checkmark$ & $\checkmark$ & $x$ & $x$ & $x$ & $x$ & $x$ & $x$ \\
\hline Personalized Cancer Therapy & Gene & $\begin{array}{l}-30 \text { known cancer } \\
\text { genes }\end{array}$ & $\checkmark$ & $x$ & $x$ & $x$ & $x$ & $x$ & $x$ & $x$ & $x$ \\
\hline JAX-CKB & Gene, Variant & $\begin{array}{l}82 \text { known cancer } \\
\text { driver genes }\end{array}$ & $\checkmark$ & $x$ & $x$ & $x$ & $x$ & $x$ & $x$ & $x$ & $x$ \\
\hline Рмкв & Gene, Variant & $\begin{array}{l}605 \text { known cancer } \\
\text { genes }\end{array}$ & $\checkmark$ & $x$ & $\checkmark$ & $x$ & $x$ & $x$ & $x$ & $x$ & $x$ \\
\hline CancerResource & $\begin{array}{l}\text { Gene, } \\
\text { pathway }\end{array}$ & $\begin{array}{l}>3000 \text { cancer } \\
\text { relevant genes }\end{array}$ & $x$ & $x$ & $x$ & $x$ & $x$ & $x$ & $x$ & $x$ & $x$ \\
\hline
\end{tabular}

b

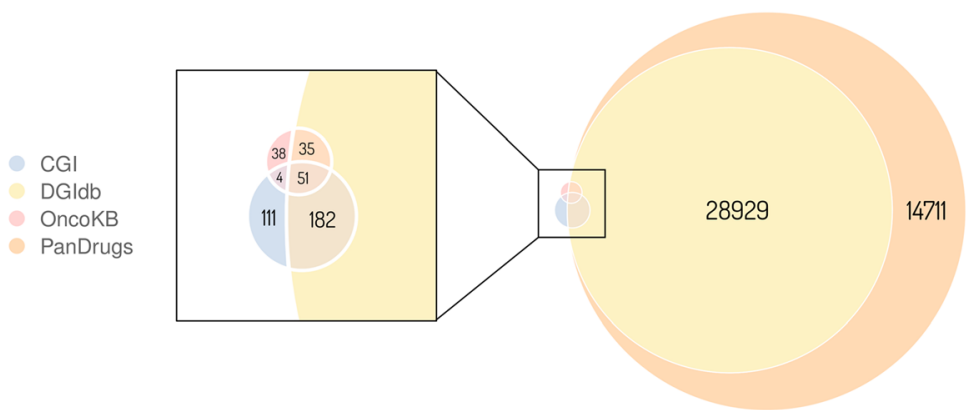

Fig. 3 a Comparison of current in silico drug prescription tools based on genomic data. b Venn diagram for drug-gene associations available in DGldb v3.0.2, Cancer Genome Interpreter, OncoKB, and PanDrugs. Global data for associations from CancerResource and Personalized Cancer Therapy is not accessible. Total numbers for non-redundant drug-gene interactions after drug standardization using PubChem to compare the resources are 29,197 (DGidb), 349 (CGI), 129 (OncoKB), and 43,909 (PanDrugs)

currently targeted with approved drugs although STAT3 inhibitors are under clinical trial. PanDrugs was queried with the 32 damaged genes and proposed, among others, Dabrafenib (LIMK1 as the direct target) and Paclitaxel (CDK5R1 as the pathway member).

In the TP53-deficient breast cancer patient (DO5375) with 15 damaged genes detected (none of them known drivers), Vismodegib, an inhibitor of the Hedgehog signaling pathway, is proposed by PanDrugs as best therapeutic candidate driven by $L R P 2$, a damaged gene that belongs to this pathway. Paclitaxel and Doxorubicin are also proposed by PanDrugs as the best therapeutic candidates. Interestingly, the combination of Vismodegib plus Paclitaxel and Epirubicin (an analog of Doxorubicin) is currently under clinical trial as neoadjuvant chemotherapy in triple negative breast cancer patients [https://clinicaltrials.gov/ct2/show/NCT02694224]. Eight non-driver damaged genes were found in the prostate cancer case (DO46813). Currently these genes have no drugs available to directly target them. Here, PanDrugs assigns the best DScores to approved MEK inhibitors and Vinblastine, an antitumoral alkaloid, to target pathway members downstream to the damaged TRAF2 gene. These examples highlight PanDrugs' capability for proposing drugs used in clinical practice in those cases with no known driver mutations and limited molecular evidence.

\section{PanDrugs application in a cancer case study}

Unfortunately, detailed clinical annotations for patients in cancer genomics international consortiums are not publicly available to validate PanDrugs results. To overcome this limitation, we have experimentally validated PanDrugs results using a patient-derived cancer mouse xenograft (PDX) model on which several therapeutic strategies have been tested as part of a personalized medicine protocol. The protocol includes whole exome sequencing analysis and the development of PDX models as described elsewhere [51].

For this validation, our case study was a 58-year-old man diagnosed with advanced squamous cell lung carcinoma (SCLC; stage IV with brain metastasis). After surgery (R0), he received a first-line chemotherapy with carboplatin/Paclitaxel. Pemetrexed/Erlotinib was administered as a second-line therapy to treat the progression of the disease.

Tumor and normal samples of this patient were sequenced to identify tumor-specific sequence alterations. We found 965 somatic mutations and 501 somatic copy number alterations (389 gain regions and 112 loss regions) (Additional file 1: Figure S11A). We detected 318 genes that would have proteins classified as damaged by mutation consequences such as stop gains, frameshifts, and deleterious missense variants. Additional file 1: Table S8 summarizes the 46 gene mutations predicted as deleterious. 
The patient's variant list (e.g. the complete VCF file) was evaluated by PanDrugs to identify druggable genomic alterations. MAPK pathway inhibitors were suggested as the best candidates. (Additional file 1: Figure $\mathrm{S} 11 \mathrm{~B})$. Indeed, likely underlying this suggestion, examination of the genomic events in this patient revealed deleterious somatic mutations in HRAS (G13 V), NF1 (K297*), and RAF1 (M562I) proteins. These mutations are predicted as damaging and may produce an activation of MAPK/ERK pathway. Constitutive activation of this pathway has been associated with cancers of the lung, colon, melanoma, lung, thyroid, leukemia, and pancreas [52] what makes it a suitable target to treat these tumours. MAPK inhibitors include compounds targeting MAP2K1 (MEK). Also, MAPK activity can still occur as a result of PI3K activation through RAS. Dual activation of these two pathways is observed in a number of cancer types including melanoma, prostate, and colorectal cancer, and provides the rationale for combining therapeutic agents [53].

We then performed an in vivo evaluation of the efficacy of several targeted antitumor agents-PI3K inhibitor (PI3Ki), MEK inhibitor (MEKi), rapamycin, dasatinib, and lapatinib-in a low passage PDX mouse model for this SCLC patient. Statistically significant $(p<0.05)$ tumor growth inhibition was reported for MEKi and PI3Ki treatments compared with the control group at the time point considered. Overall, benefit was reported with the combination of MEKi and PI3Ki towards the avatar model tested (Additional file 1: Figure S11C).

\section{Discussion}

Precision oncology requires novel resources and tools to translate cancer genomic landscapes to clinical utility in order to prescribe rational, efficient, and tailored treatments to individual cancer patients [54]. The PanDrugs method has been implemented to address the interpretation gap between raw genomic data and clinical usefulness. To this end, our methodology relies on PanDrugsdb, the largest catalogue of drug-target associations currently available. This database is publicly accessible and relates druggable genes to already approved treatments, well-known targeted therapies, and preclinical drugs.

Starting from user-supplied gene or variant lists, PanDrugs identifies and prioritizes both direct or indirect targetable genomic alterations in tumors using a novel approach based on two scores: GScore and DScore. The GScore calculates target suitability for each variant (or gene) by considering its essentiality using RNAi experimental data from the Achilles project, gene relevance in cancer, tumor frequency, and the biological and clinical impacts. The DScore evaluates drug applicability by considering its clinical indications, drug status, collective gene impact, and druggability (e.g. direct target, biomarker, or pathway member) for the genes under assessment. In addition, the DScore calculation integrates in vitro drug-screening data from GDSC and CTRP. GScore and DScore are finally evaluated together to create a gene-drug ranking offering personalized candidate treatments for the variant input list. This approach estimates the treatment adequacy based on the gene-drug associations covering more biomedical sources than any other current in silico prescription tool.

Unfortunately, current anticancer therapies are based on single biomarkers that do not consider the mutational landscape of the tumor and intratumoral clonal heterogeneity [55]. Additionally, cancer genomics studies have clearly revealed that tumoral survival and progression are mainly activated by accumulation of genetic alterations in crucial molecular pathways rather than driven by single gene alterations $[56,57]$. Thus, an accurate assessment of tumor fingerprints is essential for the development of effective therapies taking into account the collective gene impact and pathway context of genomic alterations from cancer patients $[58,59]$. Our methodology evaluates the collective gene impact by assigning a higher DScore to that drug capable to target the highest number of genes found in the input list. PanDrugs also provides prioritized treatments beyond single direct targets and biomarkers found in variant lists by exploiting the context of pathway members. Following the druggable genome paradigm PanDrugs offers a systems biology knowledge-based layer that automatically inspects biological circuits. Interestingly, this expands cancer candidate therapies from beyond limited cancer-related gene lists to the whole druggable pathway. To our knowledge, there is no other current tool with similar characteristics.

PanDrugs-assisted therapeutic strategies have been systematically applied to large patient cohorts using TCGA patients. The feasibility of our candidate treatment proposals has been also tested in PDX experimental models. In these analyses, we found that the pathway member paradigm is able to expand in silico drug prescriptions for already approved drugs. This might have a direct impact on improving clinical decision-making by extending treatment opportunities to those patients without a clear approved pharmacogenomics biomarker.

The TCGA analysis was carried out establishing a compromise GScore threshold to retrieve highly reliable candidate treatments for well-known genes. This strategy avoids handling huge lists of results by preserving best candidates, but also discards drug-gene associations found in poorly annotated genes. However, Martincorena et al. have recently reported that half of the coding driver mutations occur outside of known cancer driver genes [7]. If this is true, precision oncology will demand the implementation of novel methodologies capable of prescribing therapies beyond known cancer genes. 
PanDrugs offers novel therapeutic strategies for such genes; lowering the GScore threshold while keeping the default DScore cut-off is enough to uncover reliable therapeutic options that can target poorly annotated genes. This would allow the discovery of novel clinically significant and actionable mutations that could become new genetic predictive and prognostic biomarkers.

It is important to remark that PanDrugs is more than an organized catalogue of known gene-drug relationships. PanDrugs is the first method to systematically infer novel targeted treatments following a rational framework supported by multi-gene markers, molecular pathway context, and pharmacological evidence. Our results show that in silico prescription approaches focused uniquely on known cancer genes should be complemented by incorporating drug information associated to genomic alterations located in non-cancer genes. Our approach extends the treatment opportunities of cancer patients by enriching the therapeutic arsenal against tumors and opens new avenues for personalized medicine.

Although PanDrugs offers a valuable methodology for in silico prescription, further efforts are required to improve cancer treatment by proposing more effective drugs and anticipating drug resistance. Since drug efficacy substantially depends on tissue-, cell-, and molecular-specific context [60], precision oncology tools should integrate data beyond pure genomics [61] including the combination of high-throughput drug screenings and functional experiments to unravel heterogeneous multi-omic dependencies influencing response to therapy [62, 63]. In addition, the integration of additional biological relationship layers such as protein interaction networks [64], transcriptional regulatory modules [65], or pathway activity footprints [66] should improve drug prioritization and will help to propose alternative therapeutic strategies. It is also crucial to have a comprehensive and well-structured drug ontology available that provides drug annotations (i.e. drug indication, mechanisms of action, chemical structure, side effects, drug-target associations, and drug families) for a more accurate drug prescription [67].

Finally, it should be emphasized that current in silico drug prescription tools are limited by the lack of large longitudinal precision medicine studies with accessible clinical records. Such studies are crucial to assess and validate drug proposals and refine in silico prescription algorithms to consider additional factors such as mode of drug administration, combinatorial therapies, drug repositioning, and side effects.

\section{Conclusions}

PanDrugs provides a feasible method to guide genomic-hypothesis therapies as well as to prioritize multiple druggable alterations in genomically complex tumors. Indeed, PanDrugs represents the first drug prescription tool that proposes cancer therapies with a rationale based on pathway context, collective gene impact, and information provided by functional experiments. PanDrugs has demonstrated its adaptability by being systematically applied to large cohorts of patients and by providing candidate treatments directed to druggable genes beyond cancer driver genes. Overall, our method highlights new areas of opportunity for advancing precision cancer medicine, providing a novel and fully accessible method that could be useful in decreasing the complexity of the interpretation of genomic data and clinical decision-making. PanDrugs is freely available at http://www.pandrugs.org.

\section{Availability and requirements}

Project name: PanDrugs.

Project home page: http://www.pandrugs.org

Operating system(s): Platform independent.

Programming language: Java, MySQL RDBMS, Perl.

Other requirements: Chrome, Firefox, Safari.

License: GPLv3.

\section{Additional file}

Additional file 1: Supplementary materials and methods, Figures S1-S11 and Tables S1-S8. (PDF $14845 \mathrm{~kb}$ )

\section{Abbreviations \\ CGl: Cancer Genome Interpreter; CR: CancerResource; NSCLC: Non-small cell lung cancer; PCT: Personalized cancer therapy; PDX: Patient-derived xenograft; PMKB: Precision Medicine Knowledgebase; RNAi: RNA interference; SCLC: Small-cell lung cancer; TCGA: The Cancer Genome Atlas; TKI: Tyrosine kinase inhibitor; VCF: Variant call format}

\section{Acknowledgements}

The authors thank Joaquín Dopazo, Patricia León, and José Carbonell for kindly providing the modelled pathways employed in PanDrugs implementation; and Michael Tress for his helpful comments and suggestions in the earlier version of the manuscript.

\section{Funding}

Part of our own work has been supported by a Marie-Curie Career Integration Grant (CIG) CIG334361. KT and JP-P are supported by a Severo Ochoa FPI grant doctoral fellowship by the Spanish Ministry of Economy and Competitiveness. MR-J and DG-P are supported by the Biomedical Research Centre ("Centro Singular de Galicia," www.cinbio.es) funded by "Consellería de Cultura, Educación e Ordenación Universitaria," "Xunta de Galicia," and FEDER (European Union) and by the CITI (Centro de Investigación Transferencia e Innovación) from the University of Vigo. JMR was supported by the Spanish National Institute of Bioinformatics (www.inab.org), a platform of the "Instituto de Salud Carlos III" [INB-ISCIII, PRB2].

Availability of data and materials

The webtool is freely accessible at http://www.pandrugs.org and through its programmatic API or docker image.

Authors' contributions

EP-Y, MR-J, DG-P, KT, and JMR designed PanDrugsdb, developed the computational framework, and continue to maintain PanDrugs; EP-Y and MR-J led and executed the benchmarking analyses with input from JP-P, HT, JC, GG-L, and $\mathrm{MH}$ and supervision by DG-P and FA; PPL-C and MH carried out the 
experimental validation in PDX models; TS and JC provide fruitful discussion and valuable suggestions to improve the methodology; GG-L and FA designed the study and wrote the manuscript. FA initiated and led the study. All authors read and approved the final manuscript.

\section{Ethics approval and consent to participate}

Animals were maintained at the Spanish National Cancer Research Centre (CNIO) Animal Facility (awarded with the AAALAC accreditation) in accordance with the guidelines stated in the International Guiding Principles for Biomedical Research Involving Animals, developed by the Council for International Organizations of Medical Sciences (CIOMS). Four- to six-weekold female athymic nude-Foxn1nu (nu/nu) mice were purchased from Envigo (Barcelona, Spain). All animal experiments were approved by the Competent Authority of Comunidad de Madrid (project PROEX 104/16). Patients provided written informed consent before study enrollment. The inform consent form was reviewed and approved by the IRB of Hospital de Madrid in accordance with the guiding principles of the Declaration of Helsinki.

\section{Competing interests}

The authors declare that they have no competing interests.

\section{Publisher's Note}

Springer Nature remains neutral with regard to jurisdictional claims in published maps and institutional affiliations.

\section{Author details \\ ${ }^{1}$ Spanish National Cancer Research Centre (CNIO), 3rd Melchor Fernandez Almagro st., E-28029 Madrid, Spain. ${ }^{2}$ Computer Science Department - University of Vigo, Vigo, Spain. ${ }^{3}$ Biomedical Research Centre (CINBIO), Vigo, Spain. ${ }^{4}$ Spanish National Bioinformatics Institute (INB), Madrid, Spain. ${ }^{5}$ Department of Physiology - University of Valencia, Valencia, Spain. ${ }^{6}$ Loyola University Chicago Stritch School of Medicine, Maywood, IL, USA. ${ }^{7}$ Beth Israel Deaconess Medical Center, Boston, USA.}

\section{Received: 28 December 2017 Accepted: 4 May 2018} Published online: 31 May 2018

\section{References}

1. Kamalakaran S, Varadan V, Janevski A, Banerjee N, Tuck D, McCombie WR, et al. Translating next generation sequencing to practice: opportunities and necessary steps. Mol Oncol. 2013;7(4):743-55.

2. Lyon GJ, Wang K. Identifying disease mutations in genomic medicine settings: current challenges and how to accelerate progress. Genome Med. 2012:4:7-58.

3. Andre F, Mardis E, Salm M, Soria JC, Siu LL, Swanton C. Prioritizing targets for precision cancer medicine. Ann Oncol. 2014;25:2295-303.

4. Hyman DM, Taylor BS, Baselga J. Implementing genome-driven oncology. Cell. 2017;168(4):584-99.

5. Vogelstein B, Papadopoulos N, Velculescu VE, Zhou S, Diaz LA Jr, Kinzler KW. Cancer genome landscapes. Science. 2013;339(6127):1546-58.

6. Van Allen EM, Wagle N, Stojanov P, Perrin DL, Cibulskis K, Marlow S, et al. Whole-exome sequencing and clinical interpretation of formalin-fixed, paraffin-embedded tumor samples to guide precision cancer medicine. Nat Med. 2014;20(6):682-8

7. Martincorena I, Raine KM, Gerstung M, Dawson K, Haase K, Van Loo P, et al. Universal patterns of selection in cancer and somatic tissues. Cell. 2017; 171(5):1029-41.

8. Cheng J, Demeulemeester J, Wedge DC, Vollan HKM, Pitt JJ, Russnes HG, et al. Pan-cancer analysis of homozygous deletions in primary tumours uncovers rare tumour suppressors. Nat Commun. 2017;1:1221.

9. Peterson TA, Gauran IIM, Park J, Park D, Kann MG. Oncodomains: a protein domain-centric framework for analyzing rare variants in tumor samples. PLoS Comput Biol. 2017;4:e1005428. https://doi.org/10.1371/journal.pcbi.1005428

10. Wei CH, Phan L, Feltz J, Maiti R, Hefferon T, Lu Z. tmVar 2.0: integrating genomic variant information from literature with dbSNP and ClinVar for precision medicine. Bioinformatics. 2018;34:80-7. https://doi.org/10.1093/ bioinformatics/bt×541

11. Yang H, Robinson PN, Wang K. Phenolyzer: phenotype-based prioritization of candidate genes for human diseases. Nat Methods. 2015;12(9):841-3.

12. Ghosh R, Oak N, Plon SE. Evaluation of in silico algorithms for use with ACMG/ AMP clinical variant interpretation guidelines. Genome Biol. 2017;18:225.
13. Eilbeck K, Quinlan A, Yandell M. Settling the score: variant prioritization and Mendelian disease. Nat Rev Genet. 2017;18(10):599-612. https://doi.org/10. 1038/nrg.2017.52

14. Griffith M, Griffith OL, Coffman AC, Weible JV, McMichael JF, Spies NC, et al. DGIdb 2.0: mining clinically relevant drug-gene interactions. Nat Methods. 2013;10:1209-10.

15. Chakravarty D, Gao J, Phillips SM, Kundra R, Zhang H, Wang J, et al. OncoKB: A Precision Oncology Knowledge Base. JCO Precis Oncol. 2017; https://doi. org/10.1200/PO.17.00011

16. Tamborero D, Rubio-Perez C, Deu-Pons J, Schroeder MP, Vivancos A, Rovira A, et al. Cancer Genome Interpreter annotates the biological and clinical relevance of tumor alterations. Genome Med. 2018;10:25. https://doi.org/10. $1101 / 140475$

17. Amadoz A, Sebastian-Leon P, Vidal E, Salavert F, Dopazo J. Using activation status of signaling pathways as mechanism-based biomarkers to predict drug sensitivity. Sci Rep. 2015;5:18494. https://doi.org/10.1038/srep18494

18. Garnett MJ, Edelman EJ, Heidorn SJ, Greenman CD, Dastur A, Lau KW, et al. Systematic identification of genomic markers of drug sensitivity in cancer cells. Nature. 2012;483(7391):570-5.

19. Basu A, Bodycombe NE, Cheah JH, Price EV, Liu K, Schaefer Gl, et al. An interactive resource to identify cancer genetic and lineage dependencies targeted by small molecules. Cell. 2013;154:1151-61.

20. lorio F, Knijnenburg TA, Vis DJ, Bignell GR, Menden MP, Schubert M, et al. A landscape of pharmacogenomic interactions in cancer. Cell. 2016;166(3):740-54.

21. Bollag G, Tsai J, Zhang J, Zhang C, Ibrahim P, Nolop K, et al. Vemurafenib: the first drug approved for BRAF-mutant cancer. Nat Rev Drug Discov. 2012; 11(11):873-86.

22. Bryant HE, Schultz N, Thomas HD, Parker KM, Flower D, Lopez E, et al. Specific killing of BRCA2-deficient tumours with inhibitors of poly(ADPribose) polymerase. Nature. 2005;434(7035):913-7.

23. De Roock W, De Vriendt V, Normanno N, Ciardiello F, Tejpar S. KRAS, BRAF, PIK3CA, and PTEN mutations: implications for targeted therapies in metastatic colorectal cancer. Lancet Oncol. 2011;12(6):594-603.

24. Iyer G, Hanrahan AJ, Milowsky MI, Al-Ahmadie H, Scott SN, Janakiraman M. Genome sequencing identifies a basis for everolimus sensitivity. Science. 2012;338(6104):221.

25. Perini GF, Campregher PV, Ross JS, Ali S, Hamerschlak N, Santos FPS. Clinical response to everolimus in a patient with Hodgkin's lymphoma harboring a TSC2 mutation. Blood Cancer J. 2016;6(e420) https://doi.org/10.1038/bcj.2016.25.

26. Faber AC, Li D, Song Y, Liang MC, Yeap BY, Bronson RT, et al. Differential induction of apoptosis in HER2 and EGFR addicted cancers following PI3K inhibition. Proc Natl Acad Sci U S A. 2009;106(46):19503-8.

27. Zhu Z, Aref AR, Cohoon TJ, Barbie TU, Imamura Y, Yang S, et al. Inhibition of KRAS-driven tumorigenicity by interruption of an autocrine cytokine circuit. Cancer Discov. 2014:4(4):452-65.

28. Butler DE, Marlein C, Walker HF, Frame FM, Mann VM, Simms MS, et al. Inhibition of the PI3K/AKT/mTOR pathway activates autophagy and compensatory Ras/Raf/MEK/ERK signalling in prostate cancer. Oncotarget. 2017:8(34):56698-713.

29. Lee HJ, Zhuang G, Cao Y, Du P, Kim HJ, Settleman J. Drug resistance via feedback activation of Stat3 in oncogene-addicted cancer cells. Cancer Cell. 2014;26(2):207-21

30. Forbes SA, Beare D, Boutselakis H, Bamford S, Bindal N, Tate J, et al. COSMIC: somatic cancer genetics at high-resolution. Nucleic Acids Res. 2017;45(D1): D777-83.

31. Lawrence MS, Stojanov P, Mermel CH, Robinson JT, Garraway LA, Golub TR, et al. Discovery and saturation analysis of cancer genes across 21 tumour types. Nature. 2014;505(7484):495-501. https://doi.org/10.1038/nature12912

32. Tamborero D, Gonzalez-Perez A, Perez-Llamas C, Deu-Pons J, Kandoth C, Reimand J, et al. Comprehensive identification of mutational cancer driver genes across 12 tumor types. Sci Rep. 2013;3:2650.

33. Schlicker A, Michaut M, Rahman R, Wessels LF. OncoScape: Exploring the cancer aberration landscape by genomic data fusion. Sci Rep. 2016:6:28103.

34. Cowley GS, Weir BA, Vazquez F, Tamayo P, Scott JA, Rusin S, et al. Parallel genome-scale loss of function screens in 216 cancer cell lines for the identification of context-specific genetic dependencies. Sci Data. 2014;1: 140035 .

35. Shao DD, Tsherniak A, Gopal S, Weir BA, Tamayo P, Stransky N, et al. ATARiS: computational quantification of gene suppression phenotypes from multisample RNAi screens. Genome Res. 2013;23(4):665-78. 
36. Salavert F, Hidalgo MR, Amadoz A, Çubuk C, Medina I, Crespo D, et al. Actionable pathways: interactive discovery of therapeutic targets using signaling pathway models. Nucleic Acids Res. 2016;44(W1):W212-6.

37. Workman P, Al-Lazikani B. Drugging cancer genomes. Nat Rev Drug Discov. 2013;12(12):889-90.

38. Editorial. It's all druggable. Nat Genet. 2017;49(2):169. https://doi.org/10. 1038/ng.3788.

39. Engelman JA, Zejnullahu K, Mitsudomi T, Song Y, Hyland C, Park J, et al. MET amplification leads to gefitinib resistance in lung cancer by activating ERBB3 signaling. Science. 2007;316(5827):1039-43.

40. Turke AB, Zejnullahu K, Wu YL, Song Y, Dias-Santagata D, Lifshits E, et al. Preexistence and clonal selection of MET amplification in EGFR mutant NSCLC. Cancer Cell. 2010;17(1):77-88.

41. Gainor JF, Niederst MJ, Lennerz JK, Dagogo-Jack I, Stevens S, Shaw AT, et al. Dramatic response to combination erlotinib and crizotinib in a patient with advanced, EGFR-mutant lung cancer harboring De Novo MET amplification. J Thorac Oncol. 2016:11(7):83-5.

42. Tricker EM, Xu C, Uddin S, Capelletti M, Ercan D, Ogino A, et al. Combined EGFR/MEK inhibition prevents the emergence of resistance in EGFR-Mutant lung cancer. Cancer Discov. 2015;5(9):960-71.

43. Ortiz-Cuaran S, Scheffler M, Plenker D, Dahmen L, Scheel AH, Fernandez-Cuesta $L$, et al. Heterogeneous mechanisms of primary and acquired resistance to third-generation EGFR inhibitors. Clin Cancer Res. 2016;22(19):4837-47.

44. Kandoth C, McLellan MD, Vandin F, Ye K, Niu B, Lu C, et al. Mutational landscape and significance across 12 major cancer types. Nature. 2013; 502(7471):333-9. https://doi.org/10.1038/nature12634

45. Futreal PA, Coin L, Marshall M, Down T, Hubbard T, Wooster R, et al. A census of human cancer genes. Nat Rev Cancer. 2004:4(3):177-83.

46. Dienstmann R, Jang IS, Bot B, Friend S, Guinney J. Database of genomic biomarkers for cancer drugs and clinical targetability in solid tumors. Cancer Discov. 2015;5(2):118-23.

47. Rubio-Perez C, Tamborero D, Schroeder MP, Antolín AA, Deu-Pons J, PerezLlamas C, et al. In silico prescription of anticancer drugs to cohorts of 28 tumor types reveals targeting opportunities. Cancer Cell. 2015;27(3):382-96.

48. Gohlke BO, Nickel J, Otto R, Dunkel M, Preissner R. CancerResource-updated database of cancer-relevant proteins, mutations and interacting drugs. Nucleic Acids Res. 2016;44(D1):D932-7.

49. Patterson SE, Liu R, Statz CM, Durkin D, Lakshminarayana A, Mockus SM. The clinical trial landscape in oncology and connectivity of somatic mutational profiles to targeted therapies. Hum Genomics. 2016;10:4.

50. Goldman M, Zhang J, Fonseca NA, Xiang Q, Craft B, Piñeiro-Yáñez E, et al. Online resources for PCAWG data exploration, visualization, and discovery. https://www.biorxiv.org/content/early/2017/10/20/163907

51. Garralda E, Paz K, López-Casas PP, Jones S, Katz A, Kann LM, et al. Integrated next-generation sequencing and avatar mouse models for personalized cancer treatment. Clin Cancer Res. 2014;20(9):2476-84.

52. Dhillon AS, Hagan S, Rath O, Kolch W. MAP kinase signalling pathways in cancer. Oncogene. 2007;26(22):3279-90.

53. Mendoza MC, Er EE, Blenis J. The Ras-ERK and PI3K-mTOR pathways: crosstalk and compensation. Trends Biochem Sci. 2011;36(6):320-8.

54. Gómez-López G, Dopazo J, Cigudosa JC, Valencia A, Al-Shahrour F. Precision medicine needs pioneering clinical bioinformaticians. Brief Bioinform. 2017; https://doi.org/10.1093/bib/bbx144.

55. Dagogo-Jack I, Shaw AT. Tumour heterogeneity and resistance to cancer therapies. Nat Rev Clin Oncol. 2018:15, 81-94. https://doi.org/10.1038/ nrclinonc.2017.166

56. Garraway LA, Verweij J, Ballman KV. Precision oncology: an overview. J Clin Oncol. 2013;31(15):1803-5.

57. Califano A, Alvarez MJ. The recurrent architecture of tumour initiation, progression and drug sensitivity. Nat Rev Cancer. 2017;17(2):116-30.

58. Naulaerts S, Dang CC, Ballester PJ. Precision and recall oncology: combining multiple gene mutations for improved identification of drug-sensitive tumours. Oncotarget. 2017:8:97025-40.

59. Schneider G, Schmidt-Supprian M, Rad R, Saur D. Tissue-specific tumorigenesis: context matters. Nat Rev Cancer. 2017;17(4):239-53. https://doi.org/10.1038/nrc.2017.5.

60. Mina M, Raynaud F, Tavernari D, Battistello E, Sungalee S, Saghafinia S, et al. Conditional selection of genomic alterations dictates cancer evolution and oncogenic dependencies. Cancer Cell. 2017;32(2):155-68.

61. Letai A. Functional precision cancer medicine-moving beyond pure genomics. Nat Med. 2017;23(9):1028-35.
62. Tsherniak A, Vazquez F, Montgomery PG, Weir BA, Kryukov G, Cowley GS, et al. Defining a cancer dependency map. Cell. 2017;170(3):564-76.

63. McDonald ER, de Weck A, Schlabach MR, Billy E, Mavrakis KJ, Hoffman GR, et al. Project DRIVE: a compendium of cancer dependencies and synthetic lethal relationships uncovered by large-scale, deep RNAi screening. Cell. 2017;170(3):577-92.

64. Ivanov AA, Revennaugh B, Rusnak L, Gonzalez-Pecchi V, Mo X, Johns MA, et al. The OncoPPi Portal: an integrative resource to explore and prioritize protein-protein interactions for cancer target discovery. Bioinformatics. 2018; 34:1183-91. https://doi.org/10.1093/bioinformatics/btx743

65. Garcia-Alonso L, lorio F, Matchan A, Fonseca N, Jaaks P, Peat G, et al. Transcription factor activities enhance markers of drug sensitivity in cancer. Cancer Res. 2018;78:3,769-80.

66. Schubert $M$, Klinger B, Klünemann M, Sieber A, Uhlitz F, Sauer $S$, et al. Perturbation-response genes reveal signaling footprints in cancer gene expression. Nat Commun. 2018;9(1):20.

67. Sharp ME. Toward a comprehensive drug ontology: extraction of drugindication relations from diverse information sources. J Biomed Semantics. 2017:8(1):2.

\section{Ready to submit your research? Choose BMC and benefit from:}

- fast, convenient online submission

- thorough peer review by experienced researchers in your field

- rapid publication on acceptance

- support for research data, including large and complex data types

- gold Open Access which fosters wider collaboration and increased citations

- maximum visibility for your research: over $100 \mathrm{M}$ website views per year

At BMC, research is always in progress.

Learn more biomedcentral.com/submissions 